\title{
Comparing brain white matter on sequential cranial ultrasound and MRI in very preterm infants
}

\author{
Lara M. Leijser • Lishya Liauw • Sylvia Veen • \\ Inge P. de Boer • Frans J. Walther • \\ Gerda van Wezel-Meijler
}

Received: 16 July 2007 / Accepted: 30 April 2008 /Published online: 11 June 2008

(C) The Author(s) 2008

\begin{abstract}
Introduction Periventricular white matter (WM) echodensities, frequently seen in preterm infants, can be associated with suboptimal neurodevelopment. Major WM injury is well detected on cranial ultrasound (cUS). cUS seems less sensitive for diffuse or more subtle WM injury. Our aim was to assess the value of cUS and magnetic resonance imaging (MRI) for evaluating WM changes and the predictive value of cUS and/or MRI findings for neurodevelopmental outcome in very preterm infants with normal to severely abnormal WM on sequential high-quality cUS. Materials and methods Very preterm infants ( $<32$ weeks) who had sequential cUS and one MRI within the first three postnatal months were included. Periventricular WM on cUS and MRI was compared and correlated with neurodevelopmental outcome at 2 years corrected age.

Results Forty preterm infants were studied; outcome data were available in 32 . WM changes on sequential cUS were predictive of WM changes on MRI. Severely abnormal WM on cUS/MRI was predictive of adverse outcome, and normal-mildly abnormal WM of favorable outcome. Moderately abnormal WM on cUS/MRI was associated with variable outcome. Additional MRI slightly increased the predictive value of cUS in severe WM changes.
\end{abstract}

\footnotetext{
L. M. Leijser · S. Veen · I. P. de Boer · F. J. Walther •

G. van Wezel-Meijler $(\square)$

Department of Pediatrics, Division of Neonatology, Leiden University Medical Center,

Albinusdreef 2, P.O. Box 9600, 2300 RC Leiden, the Netherlands

e-mail: G.van_Wezel-Meijler@lumc.nl

L. Liauw

Department of Radiology, Division of Neuroradiology,

Leiden University Medical Center,

Albinusdreef 2, P.O. Box 9600, 2300 RC Leiden, the Netherlands
}

Conclusion Sequential cUS in preterm infants is reliable for detecting WM changes and predicting favorable and severely abnormal outcome. Conventional and diffusionweighted MRI sequences before term equivalent age in very preterm infants, suggested on cUS to have mild to moderately abnormal WM, do not seem to be warranted.

Keywords Ultrasound - White matter · MRI · Outcome . Preterm infant

$\begin{array}{ll}\text { Abbreviations } \\ \text { cUS } & \text { cranial ultrasound } \\ \text { DEHSI } & \text { diffuse and excessive high signal intensity } \\ \text { GA } & \text { gestational age } \\ \text { MDI } & \text { mental developmental index } \\ \text { MRI } & \text { magnetic resonance imaging } \\ \text { NPV } & \text { negative predictive value } \\ \text { PDI } & \text { psychomotor developmental index } \\ \text { PPV } & \text { positive predictive value } \\ \text { PVL } & \text { periventricular leukomalacia } \\ \text { SI } & \text { signal intensity } \\ \text { TEA } & \text { term equivalent age } \\ \text { WM } & \text { white matter }\end{array}$

\section{Introduction}

Cranial ultrasound (cUS) is the preferred and most readily available tool to assess the neonatal brain [1]. It is a safe and reliable technique for demonstrating the most frequently occurring forms of cerebral injury in preterm infants, assessing the evolution of lesions, and following brain development [1-7]. Magnetic resonance imaging (MRI) demonstrates site and extent of cerebral lesions more precisely and shows maturational processes in detail [3, 4, 8-10]. 
In preterm infants, increased echogenicity in the periventricular white matter (WM), so-called periventricular flaring, may represent ischemic and/or inflammatory damage. Flares are transient, persisting for a variable period of time, and can subsequently resolve or evolve into cystic lesions [2]. When persisting for over 1 week and when the echogenicity exceeds that of the choroid plexus, flares are considered the first stage of periventricular leukomalacia (PVL) [2]. Cystic forms of PVL are associated with neurological impairment and are well demonstrated by cUS $[6,11,12]$. Milder flares, not evolving into cysts, are more frequently encountered in preterm infants and, if longlasting, may also be associated with suboptimal or deviant neurological development, especially if there is change in ventricular size or shape $[2,4-6,13-16]$. However, they may also represent normal maturational phenomena in the immature brain [9, 17]. Therefore, it is important to recognize pathological flares, especially those associated with neurological sequelae.

Diffuse WM injury has been described in MRI and pathology studies in newborn infants [4, 5, 10, 12, 18-24]. Several studies have found a poor predictive value of cUS for detecting diffuse or more subtle WM injury $[4,5,7,11$, $12,18,21,23]$. It has been suggested that MRI should be performed in all very preterm infants, particularly in those with only mild (non-cystic) or no WM changes on cUS [5, $11,12,23]$. However, as MRI is more burdening to the newborn infant and more time-consuming and expensive than cUS, it is not a good tool for serial imaging of the neonatal brain, and it is important to know when MRI is warranted in sick, vulnerable preterm infants. In addition, if MRI is performed, it should be optimally timed to detect clinically significant lesions, assess brain maturation, and accurately predict outcome.

The aims of our study were to evaluate the predictive value of WM changes on sequential, high-quality cUS for those seen on MRI, to explore the additional value of MRI within the first three postnatal months for detecting WM changes, and to assess the predictive value of cUS and/or MRI findings for neurodevelopmental outcome in very preterm infants. This was done by comparing findings on sequential cUS examinations with MRI findings and by relating cUS and MRI findings to neurodevelopmental outcome at 2 years corrected age.

\section{Materials and methods}

Patients

Very preterm infants born at a gestational age (GA) of $<32$ weeks who were admitted to the neonatal intensive care unit of our tertiary neonatal referral center, between May 2001 and April 2004 and underwent at least one MRI examination during the neonatal period, were included in the study. Exclusion criteria were neonatal meningitis, metabolic disorders, chromosomal disorders, congenital cardiac abnormalities, and specific syndromes. In unstable infants with septicemia, the MRI procedure was postponed until the infant was in a stable clinical condition. Medical records, neuroimaging findings and follow-up data were reviewed.

After exclusion, data of 40 very preterm infants (31 male) were studied. Mean GA of the infants was 28.6 (range 25.1-31.9) weeks and mean birth weight 1,203 $(689-2,062) \mathrm{g}$. The mean number of cUS scans performed in each infant during admission was seven (3-16). MRI examinations were performed for various indications including very preterm birth, ventricular dilatation, (suspicion of) parenchymal injury and/or grade 2 echodensities on cUS.

Mean postnatal age and corrected GA at MRI were, respectively, 34.4 (4-111) days and 33.2 (27.3-45.1) weeks. Eight out of 40 infants were scanned at a very young postnatal age of less than 10 days and 32 out of 40 infants thereafter. In five infants, MRI was performed around term equivalent age (TEA) at a mean corrected GA of 39.4 (38.9-40.9) weeks.

\section{Cranial ultrasound}

Sequential cUS scans were performed routinely in all very preterm infants, from the day of birth until discharge or transfer to another hospital, and around TEA, according to a standard protocol. The transducer frequency was set at 7.5 MHz. For detection of cortical and/or subcortical abnormalities, higher frequencies up to $10.0 \mathrm{MHz}$ were used, whereas deeper structures were assessed with lower frequencies down to $5.0 \mathrm{MHz}$. All images were saved on Magneto-Optical Disks.

Of all included infants, the first cUS scan, the cUS scan performed closest to the day of the MRI examination and the last cUS scan before discharge were evaluated retrospectively by at least two experienced investigators (LML, Research Physician, IPdB, Neonatologist, and GvWM, Neonatologist) by consensus. The names of the infants were masked on the cUS scans, so the investigators were unaware of the MRI findings and outcome of the infants. Special attention was paid to the presence of echogenicity changes in the brain WM. For each area in the brain (frontal, parietal, occipital, and temporal) the presence and appearance of periventricular echodensities was recorded. The degree of echogenicity of the WM was scored according to the classification by van Wezel-Meijler et al. [9], which gives information on the intensity of periven- 
tricular echogenicity. A comment on homogeneity or inhomogeneity of the WM was added [10]:

Grade 0: Normal echogenicity of the periventricular WM $(<$ choroid plexus).

Grade 1: Moderately increased echogenicity of the periventricular WM, the affected region (or smaller areas within the affected region) being almost as bright or as bright as the choroid plexus. Separate notation: homogeneous, inhomogeneous.

Grade 2: Severely increased echogenicity, the affected region (or smaller areas within the affected region) being obviously brighter than the choroid plexus. Separate notation: homogeneous, inhomogeneous.

PVL was scored according to the classification by de Vries et al. [2]:

Grade 1: Transient periventricular echodensities, persisting $\geq 7$ days.

Grade 2: Transient periventricular echodensities, evolving into small, localized fronto-parietal cysts.

Grade 3: Transient periventricular echodensities, evolving into extensive periventricular cystic lesions.

Grade 4: Echodensities extending into the deep WM, evolving into extensive cystic lesions.

Peri- and intraventricular hemorrhages were classified according to Volpe [25]. The presence of other abnormalities was recorded.

The cUS scans of each infant were scored based on WM changes, i.e., grade of echogenicity and/or PVL. Subsequently, the cUS scans were classified according to the most severe changes in the WM during admission. This was done to enable comparison of the cUS and MRI findings. Sequential cUS scans without echodensities or with homogeneous grade 1 echogenicity were classified as normal [9]. cUS scans with inhomogeneous grade 1 echogenicity were classified as mildly abnormal, regardless of the total duration $[9,10]$. Scans with grade 2 echogenicity, regardless of the total duration, and/or localized, small cystic lesions (PVL grade 2) were classified as moderately abnormal, and those with multicystic PVL (PVL grades 3 and 4) and/or focal echodensities within the WM as severely abnormal $[2,9,10]$.

\section{MRI}

MR images were obtained in 40 preterm infants according to a standard MR imaging protocol for newborn infants, using a 1.5-Tesla Philips MR system (Philips Medical Systems, Best, the Netherlands). This protocol comprised $\mathrm{T}_{1^{-}}, \mathrm{T}_{2^{-}}$, and diffusion-weighted images in the axial plane (slice thickness $4-5 \mathrm{~mm}$, field of view $18-20 \mathrm{~cm}^{2}$ ), and $\mathrm{T}_{1}$ weighted images in the sagittal plane.
The infants were laid supine and snuggly swaddled-up during the scanning procedure. Ear protection consisted of neonatal earmuffs (Natus MiniMuffs; Natus Medical Inc, San Carlos, CA). The infant's head was immobilized with moulded foam, placed around the head. Temperature was maintained, and heart rate and oxygen saturation were monitored throughout the procedure. A pediatrician experienced in resuscitation and MR procedures was present during the scanning.

All MRI examinations were assessed by consensus by two experienced investigators (GvWM, Neonatologist, and LL, Pediatric Neuroradiologist). The names of the infants were masked on the MR images, so the investigators were unaware of the cUS findings and outcome of the infants. Special attention was paid to the signal intensity (SI) of brain WM. WM injury was scored according to Sie et al. [10]. This scoring system was used to rate increasingly severe changes in the periventricular WM. Whenever characteristics of more than one MRI score were present, the highest score was used. MRI scores were subdivided into different groups to enable comparison of MRI with cUS and to relate MRI to outcome. MRI examinations without WM changes (grade 1) were classified as normal, those with grade 2 and 3 changes (i.e., periventricular zone of changed SI, or $\leq 6$ punctate WM lesions) as mildly abnormal, those with grade 4 changes (i.e., $>6$ punctate WM lesions and/or small periventricular cysts and/or a few larger focal hemorrhages) as moderately abnormal, and those with grades 5 (i.e., extensive SI changes with hemorrhagic or (pre)cystic lesions in the periventricular WM, with, at most, focal subcortical extension) and 6 changes (i.e., diffuse SI changes with hemorrhagic or (pre) cystic lesions involving both the periventricular and subcortical WM) as severely abnormal. Presence of abnormalities other than WM changes was recorded. For MRI examinations performed around TEA (38-42 weeks' gestation), the presence of areas of diffuse and excessive high SI, diffusely distributed within the periventricular or subcortical WM (DEHSI) [19], was recorded on $T_{2}$ weighted MR images as a separate entity.

\section{Neurodevelopmental outcome}

Preterm infants born at a GA of $<32$ weeks and admitted to the neonatal unit of the LUMC participated in a standardized follow-up program until the corrected age of 5 years. In a total of 32 out of 40 preterm infants $(80 \%)$ with sequential cUS examinations and one or more MRI examinations outcome data were available. From 8 out of 40 infants $(20 \%)$, no outcome data at the corrected age of 24 months were available; two infants $(5 \%)$ died during the neonatal period, one because of respiratory complications, and the other because of circulatory problems, and six 
infants were lost to follow-up. The infants were assessed by neonatologists with advanced training. For the purpose of this study, neurodevelopmental outcome data of the examination closest to the corrected age of 24 months were recorded. Mean corrected age at the follow-up examination was 23.2 (22.5-26.0) months. For most infants $(n=24)$, Bayley Scales of Infant Development II scores for motor and mental development were available [26]. A Mental or Psychomotor Developmental Index (MDI, PDI) of $\geq 85$ was considered normal, a MDI or PDI between 70 and 84 as mildly abnormal, a MDI or PDI between 55 and 69 as moderately abnormal, and a MDI or PDI of $<55$ as severely abnormal. Infants of whom Bayley II scores were not available, were assigned to one of the four outcome groups based on the available data from the outpatient clinic.

For this study, normal and mildly abnormal outcome were considered as favorable outcome and moderately and severely abnormal outcome as adverse outcome.

\section{Data analysis}

Statistical analyses were performed using SPSS software (version 12.0; SPSS, Chicago, Illinois, USA). cUS and MRI findings were compared. Predictive values of cUS findings for MRI findings and cUS and/or MRI findings for neurodevelopmental outcome were calculated. For the purpose of this study, normal and mildly abnormal neuroimaging findings and normal and mildly abnormal outcome findings were considered together as one group.

\section{Results}

Cranial ultrasound $(n=40)$

In 37 out of 40 infants (93\%), periventricular echodensities were seen on sequential cUS. The echodensities persisted for over 7 days and evolved into cystic lesions in $35 \%$. In 22 out of 37 infants, the echodensities had an inhomogeneous appearance in one or more areas of the periventricular WM, while in the other infants, echodensities were consistently homogeneous. In three infants $(8 \%)$, no echodensities were seen. Twenty-three infants $(58 \%)$ had grade 1 (14 homogeneous, 9 inhomogeneous) and one (3\%) grade 2 (homogeneous) echogenicity, and seven infants (18\%) had grade 2 and six (15\%) grade 3 PVL. In 26 infants $(65 \%)$, the WM was scored as normal to mildly abnormal, in eight $(20 \%)$ as moderately abnormal, and in six $(15 \%)$ as severely abnormal.
MRI $(n=40)$

In eight out of 40 infants (20\%), no WM abnormalities were detected on MRI (grade 1). Eleven infants (28\%) showed high SI areas in the periventricular WM (grade 2). In 16 infants $(40 \%)$ punctate lesions were seen in the WM on both $\mathrm{T}_{1}$ - and $\mathrm{T}_{2}$-weighted images; in seven infants (18\%) six or less (grade 3 ) and in nine (23\%) more than six (grade 4). Four infants $(10 \%)$ had extensive SI changes in the periventricular WM with hemorrhagic and/or cystic lesions (grade 5) and one infant (3\%) had diffuse SI changes in the periventricular and subcortical WM with hemorrhagic and cystic lesions (grade 6).

In 26 infants $(65 \%)$, the WM was scored as normal to mildly abnormal, in nine $(23 \%)$ as moderately abnormal, and in five (13\%) as severely abnormal on MRI. In all five infants who underwent MRI around TEA, DEHSI was seen in the frontal and occipital WM on $\mathrm{T}_{2}$-weighted images.

Relation between cUS and MRI $(n=35)$

Twenty-four out of 35 infants had normal to mildly abnormal, six moderately abnormal, and five severely abnormal WM on both cUS and MRI. The relation between WM changes on cUS and those on MRI are depicted in Table 1. The predictive values of normal to mildly abnormal WM on cUS for normal to mildly abnormal WM on MRI were: sensitivity 0.92 , specificity 0.86 , positive predictive value (PPV) 0.92 , and negative predictive value (NPV) 0.86 ; those of moderately abnormal WM on cUS for moderately abnormal WM on MRI, respectively, 0.75, 0.91, 0.67, and 0.94; and those of severely abnormal WM on cUS for severely abnormal WM on MRI, respectively, $0.83,1.00,1.00$, and 0.97 . So, sequential cUS predicted WM changes on neonatal MRI.

In 14 out of 23 infants with grade 1 echodensities on cUS, the echodensities had a homogeneous appearance, while in 9 out of 23, they appeared inhomogeneous. In the

Table 1 Relation between WM changes on sequential cUS and on neonatal MRI within the first three postnatal months (n, number of infants)

\begin{tabular}{|c|c|c|c|}
\hline \multirow{2}{*}{$\begin{array}{l}\text { cUS findings } \\
(n=40)\end{array}$} & \multicolumn{3}{|l|}{ MRI findings } \\
\hline & $\begin{array}{l}\text { Normal-mildly } \\
\text { abnormal } \\
(n=26)\end{array}$ & $\begin{array}{l}\text { Moderately } \\
\text { abnormal } \\
(n=9)\end{array}$ & $\begin{array}{l}\text { Severely } \\
\text { abnormal } \\
(n=5)\end{array}$ \\
\hline $\begin{array}{l}\text { Normal-mildly } \\
\text { abnormal }(n=26)\end{array}$ & 24 & 2 & \\
\hline $\begin{array}{l}\text { Moderately abnormal } \\
(n=8)\end{array}$ & 2 & 6 & \\
\hline $\begin{array}{l}\text { Severely abnormal } \\
(n=6)\end{array}$ & & 1 & 5 \\
\hline
\end{tabular}


14 infants with homogeneous echodensities, MRI showed normal WM in four, a periventricular zone of changed SI in eight and punctate lesions in two. In the nine infants with inhomogeneous echodensities, MRI showed normal WM in one, a periventricular zone of changed SI in two, and punctate lesions in six. In the infant with homogeneous grade 2 echodensities, MRI showed punctate WM lesions.

In six out of seven infants with punctate WM lesions on MRI, cUS showed inhomogeneous echodensities in the periventricular WM. These were grade 1 echodensities in five infants and grade 2 echodensities in one infant.

In none of the infants, additional lesions were seen on sequential cUS after the MRI was performed, and the longest time-interval between the MRI and the last cUS examination that was scored was 10 days.

Examples of WM changes on cUS and MRI examinations of studied infants are presented in Figs. 1, 2, 3, 4, and 5.
Neurodevelopmental outcome $(n=32)$

Twenty out of 32 infants $(63 \%)$ were classified as normal to mildly abnormal, five (16\%) as moderately abnormal, and seven $(22 \%)$ as severely abnormal.

Relation between cUS and outcome $(n=32)$

The relation between cUS findings and neurodevelopmental outcome is shown in Table 2. The predictive values of normal to mildly abnormal and severely abnormal cUS findings for, respectively, normal to mildly abnormal (favorable) and severely abnormal outcome are shown in Table 3. Moderately abnormal cUS findings were associated with variable outcome. So, normal to mildly abnormal WM on cUS was predictive of normal to mildly abnormal outcome and severely abnormal WM on cUS of severely abnormal
Fig. 1 Coronal (a) and parasagittal (b) cUS scan of a preterm infant (GA 25.1 weeks) at a corrected GA of 32.0 weeks (postnatal age 48 days) showing normal-appearing brain WM (classified as normal WM). Note the symmetrical, subtle echodensities in the frontal WM that are considered a normal finding in this age group (arrow; ref. [9]) and the symmetrical echodensities in the area of the basal ganglia (stars; ref. [36]). Transverse $\mathrm{T}_{1^{-}}(\mathbf{c})$ and $\mathrm{T}_{2}$-weighted (d) MR image at the level of the basal ganglia of the same infant, performed on the same day as the cUS scans, also showing normal-appearing brain WM (classified as normal WM). Note the normal maturational phenomena of the WM, especially prominent on the $\mathrm{T}_{2}$-weighted MR image, showing bands of alternating SI within the WM (arrows; [19]). This infant had a normal outcome at 2 years corrected age
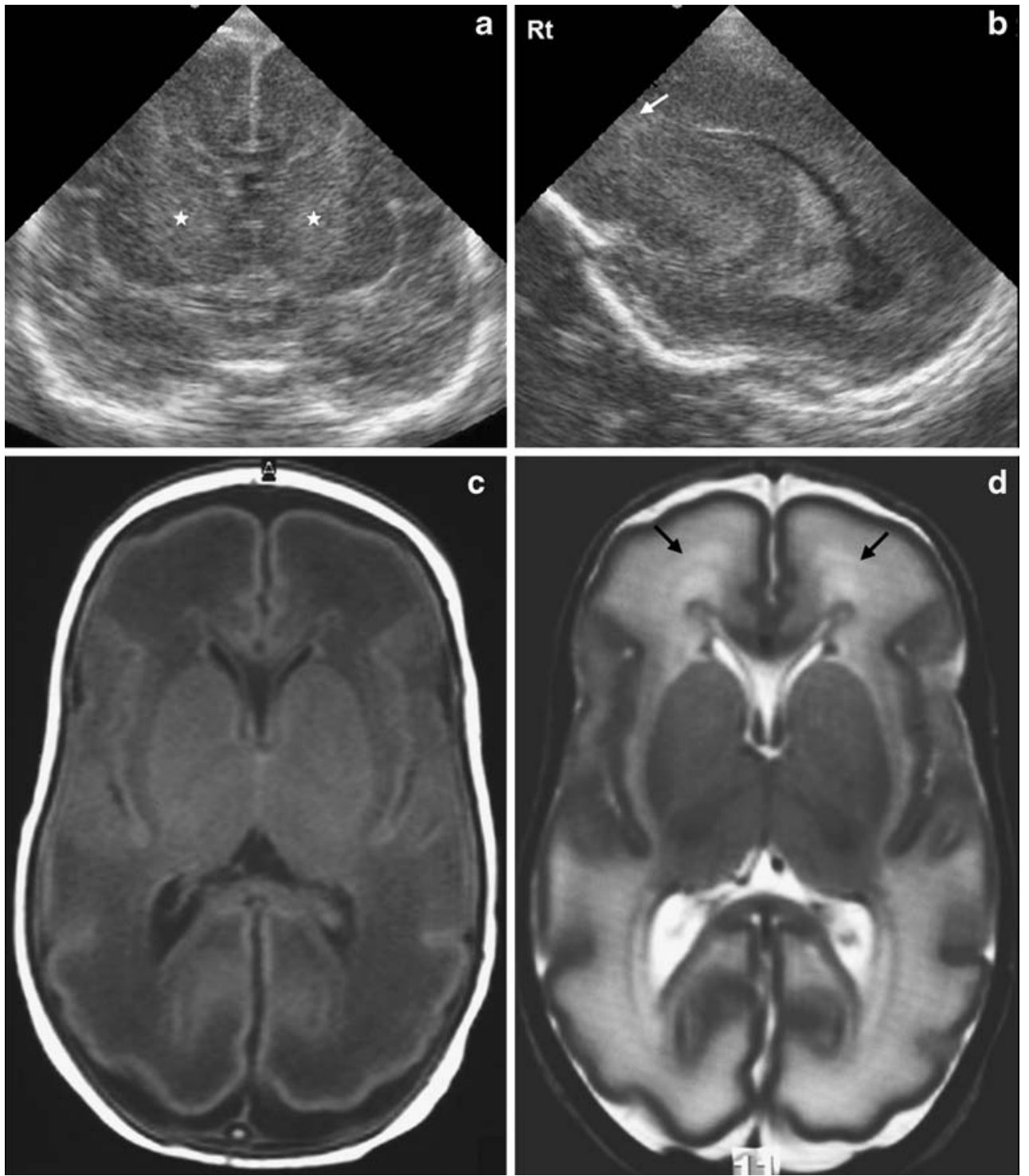
Fig. 2 Parasagittal cUS scans (a, b) of a preterm infant (GA 28.0 weeks) at a corrected GA of 32.4 weeks (postnatal age 31 days) showing mildly increased echogenicity (less than the echogenicity of the choroid plexus) in the parietal WM (arrows; classified as normal WM). cUS also demonstrated a right-sided intraventricular hemorrhage grade 2 (not shown here). Transverse $T_{1^{-}}(\mathbf{c})$ and $T_{2-}$ weighted (d) MR image at high ventricular level of the same infant, performed 3 days after the cUS scans (postnatal age 34 days), showing punctate hemorrhages $(<6)$ in the periventricular WM on the right (arrows; classified as mildly abnormal WM), an intraventricular hemorrhage on the right, and a very small germinal matrix hemorrhage on the left. This infant had a mildly abnormal outcome at 2 years corrected age
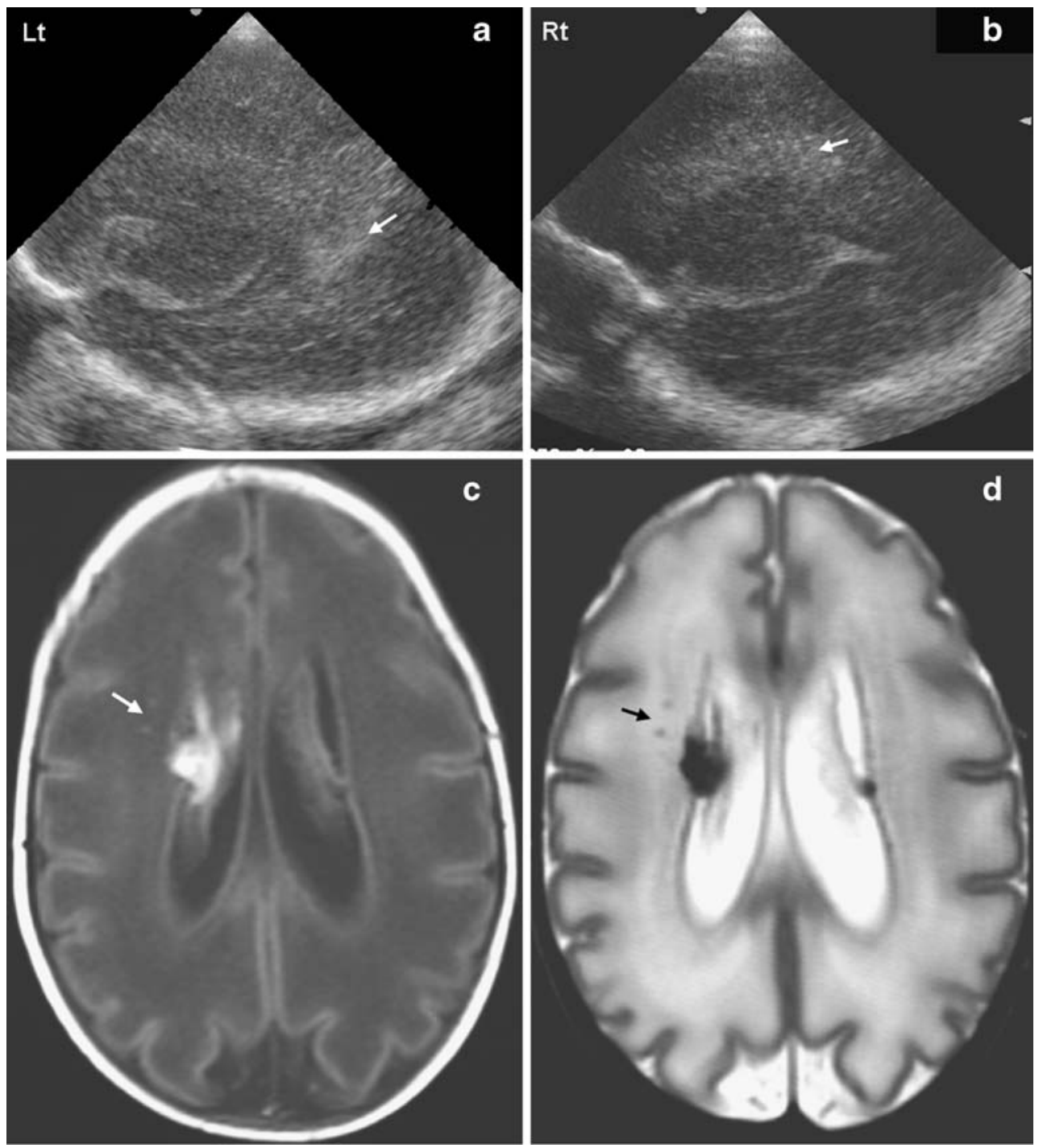

outcome, while moderately abnormal WM on cUS had low predictive values for moderately abnormal outcome.

Relation between MRI and outcome $(n=32)$

The relation between MRI findings and neurodevelopmental outcome is shown in Table 4. Like for the cUS findings, the predictive values of MRI findings for outcome are shown in Table 3. Comparable predictive values for outcome were found for the MRI findings as for the cUS findings, although severely abnormal, WM on MRI was highly predictive of severely abnormal outcome.

In 6 out of 32 infants, MRI was performed before the postnatal age of 10 days and in 26 out of 32 after 10 days. No differences were found in predictive values of MRI findings for outcome between the two subgroups.

There was no tendency for more severe WM changes (grades 4-6) occurring more often on MRI scans performed after 10 days than before 10 days.
None of the infants developed adverse events after the MRI examination that could be of importance for outcome.

Relation between cUS combined with MRI and outcome $(n=28)$

From 19 infants with normal to mildly abnormal, all six infants with moderately abnormal and three infants with severely abnormal WM on both cUS and MRI, outcome data were available. The relation between cUS and MRI findings and neurodevelopmental outcome is shown in Table 5. Like for the cUS and MRI findings separately, the predictive values of the combined cUS and MRI findings for outcome are shown in Table 3. Comparable predictive values for outcome were found for the combined cUS and MRI findings as for the cUS findings alone, although severely abnormal WM on cUS and MRI was highly predictive of severely abnormal outcome. Thus, MRI performed within the first three postnatal months slightly 
Fig. 3 Coronal (a) and parasagittal $(\mathbf{b}, \mathbf{c})$ cUS scans of a preterm infant (GA 28.1 weeks) at a corrected GA of 31.0 weeks (postnatal age 20 days) showing an intraventricular hemorrhage (long arrow) with periventricular intraparenchymal echodensity on the left (medium arrow), rather localized inhomogeneously increased echogenicity in the parietal WM on the left (short arrow; classified as severely abnormal WM), and mildly increased echogenicity in the parietal WM on the right (short arrow; classified as normal WM). Transverse $\mathrm{T}_{1^{-}}$(d) and $\mathrm{T}_{2}$-weighted (e) MR image at the level of the centrum semiovale of the same infant, performed 1 day before the cUS scans (postnatal age 19 days), showing bilateral SI changes in the parieto-occipital WM on the $\mathrm{T}_{2}$-weighted MR image (e; long arrows), possibly a normal finding at this age, and several small cystic lesions and punctate hemorrhagic lesions $(>6)$ in the parietal WM on the left on the $\mathrm{T}_{1^{-}}$(d) and $\mathrm{T}_{2}$-weighted (e) MR image (short arrows; classified as severely abnormal WM).

These abnormalities were also seen at a lower level and extended into the ipsilateral basal ganglia and internal capsule. This infant had a severely abnormal outcome at 2 years corrected age
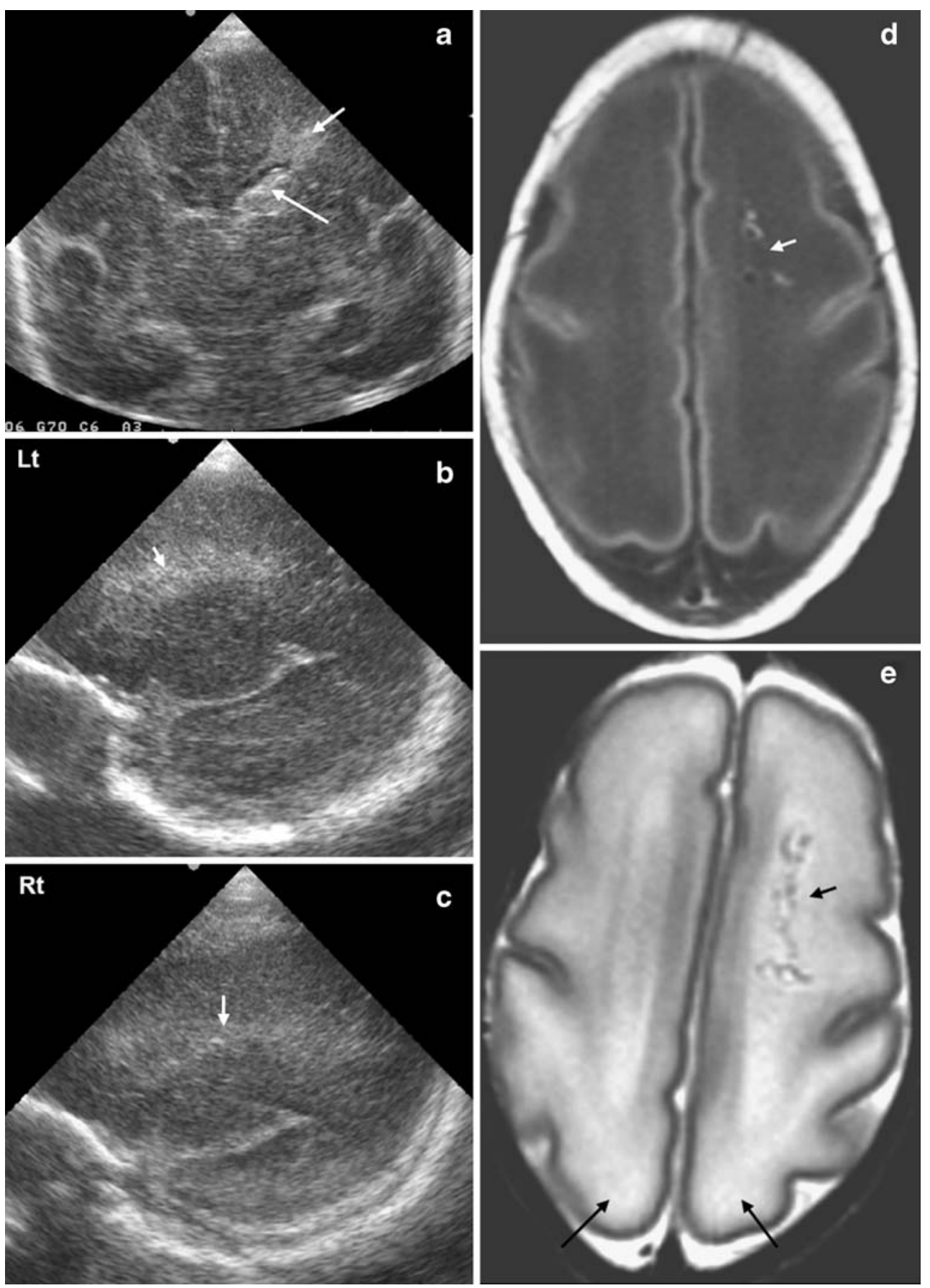

increased the predictive value of cUS for severely abnormal outcome.

Relation between DEHSI and cUS and outcome findings $(n=5)$

In all five infants in whom MRI was performed around TEA, DEHSI was present in the frontal and occipital WM. DEHSI on MRI was associated with variable other neuroimaging findings; one infant had normal WM on cUS and MRI, one mildly abnormal WM and two moderately abnormal WM, and one mildly abnormal WM on cUS and normal WM on MRI. Four of the five infants $(80.0 \%)$ with DEHSI did not have a normal outcome at 2 years corrected age.

\section{Discussion}

This study retrospectively assessed cUS and brain MRI findings within the first three postnatal months in very preterm infants, focusing on the periventricular WM. cUS 
Fig. 4 Coronal (a) and parasagittal (b, c) cUS scans of a preterm infant (GA 29.1 weeks) at a corrected GA of 33.0 weeks (postnatal age 27 days) showing inhomogeneously increased echogenicity in the parietal WM on both sides (arrows; classified as moderately abnormal WM). Note the symmetrical echodensities in the area of the basal ganglia [36]. Parasagittal $\mathrm{T}_{1}$ weighted MR image (d) through the right lateral ventricle and basal ganglia region and transverse $T_{2}$-weighted $M R$ image (e) at the level of the centrum semiovale of the same infant, performed 2 days after the cUS scans (postnatal age 29 days), showing multiple hemorrhagic lesions (>6) in the WM, being punctate on the right (short arrows) and also more extensive on the left (long arrow; classified as moderately abnormal WM). This infant had a moderately abnormal outcome at 2 years corrected age
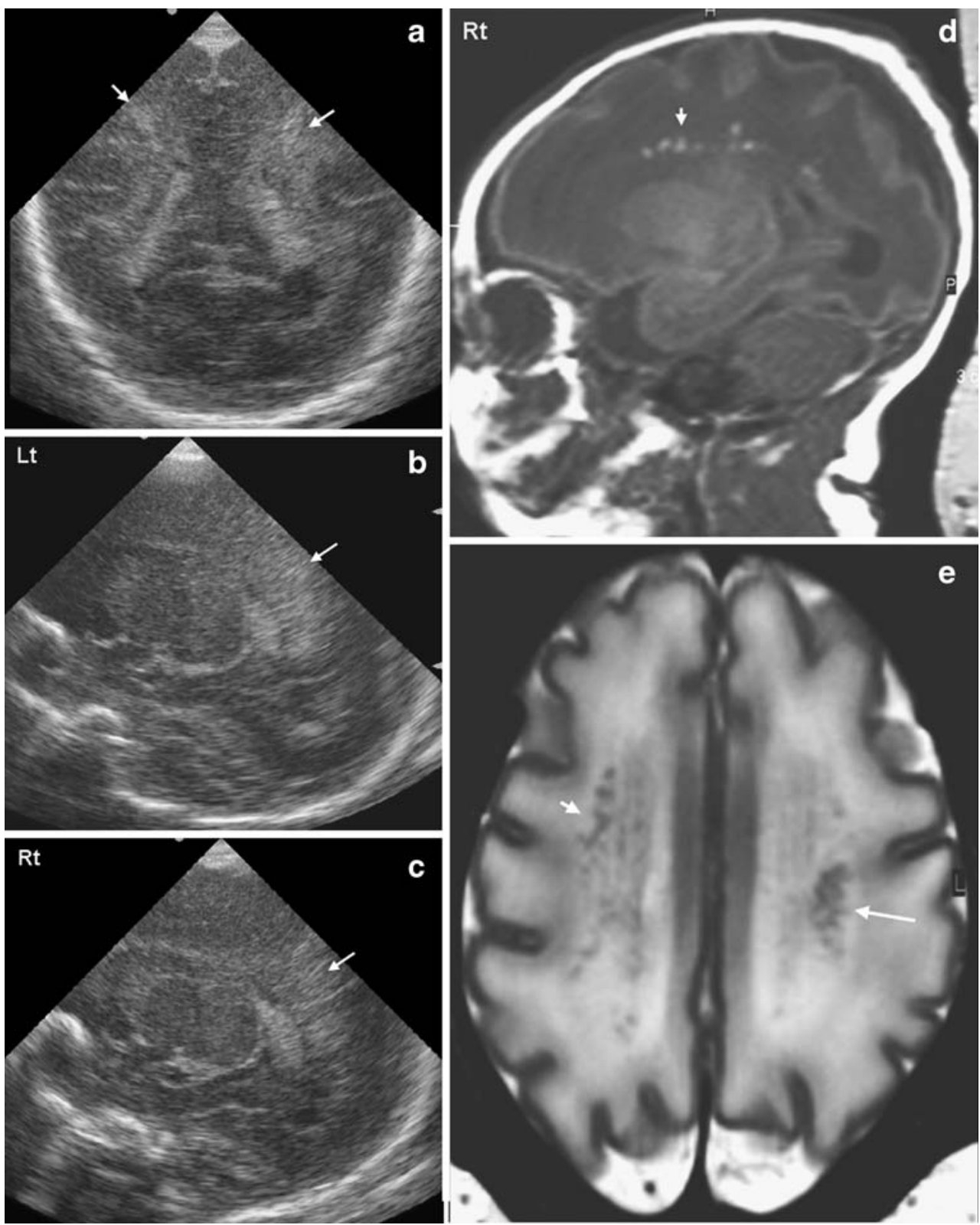

e and MRI findings were compared and related to neurodevelopmental outcome. Sequential cUS was predictive of WM changes on neonatal MRI. Our findings are partially consistent with several cUS, pathology, and MRI correlation studies in preterm infants, showing that cUS is a reliable tool for demonstrating major forms of WM lesions, including cystic PVL and parenchymal infarction $[2-5,7$, $10-12,21]$, but a poorer predictor of diffuse and more subtle WM lesions, such as punctate WM lesions [4, 5, 7, $10-12,18,21,23]$. In nearly all infants with punctate WM lesions on MRI, cUS showed inhomogeneous grade 1 echodensities in the periventricular WM. We therefore hypothesize that inhomogeneous grade 1 echodensities are the cUS correlate of punctate WM lesions. This is different from previous studies showing that in preterm infants with punctate WM lesions on MRI, no corresponding lesions are detected on cUS $[10,11,21]$ and can be explained by our performance of frequent cUS examinations throughout the neonatal period until TEA. For this study, we did not only assess the cUS performed close to the MRI examination, but assessed three cUS examinations and used the cUS with the most severe WM changes for comparison with MRI.

In the 14 infants with homogeneous grade 1 echodensities, MRI showed normal WM or a periventricular zone of changed SI in all but two infants, while in the nine infants with inhomogeneous grade 1 echodensities, MRI showed punctate lesions in six. In the infant with homogeneous grade 2 echodensities, MRI showed punctate WM lesions. These findings suggest that inhomogeneous echodensities are associated with more severe WM changes on MRI than 
Fig. 5 Coronal (a) and parasagittal (b) cUS scan of a preterm infant (GA 26.3 weeks) around TEA (corrected GA 42.0 weeks, postnatal age 105 days) showing a widened interhemispheric fissure (arrow) and normal-appearing brain $\mathrm{WM}$ (classified as normal WM). Transverse $\mathrm{T}_{2^{-}}(\mathbf{c})$ and diffusionweighted (d) MR image at high ventricular level of the same infant, performed on the same day as the cUS scans, showing a widened interhemispheric fissure in the frontal region (long arrow). Also showing DEHSI in the frontal and parieto-occipital WM on the $\mathrm{T}_{2}$-weighted image (c; short arrows), and high signal in the frontal and parietooccipital WM on the diffusionweighted image (d; short arrows). This infant had a mildly abnormal outcome at 2 years corrected age
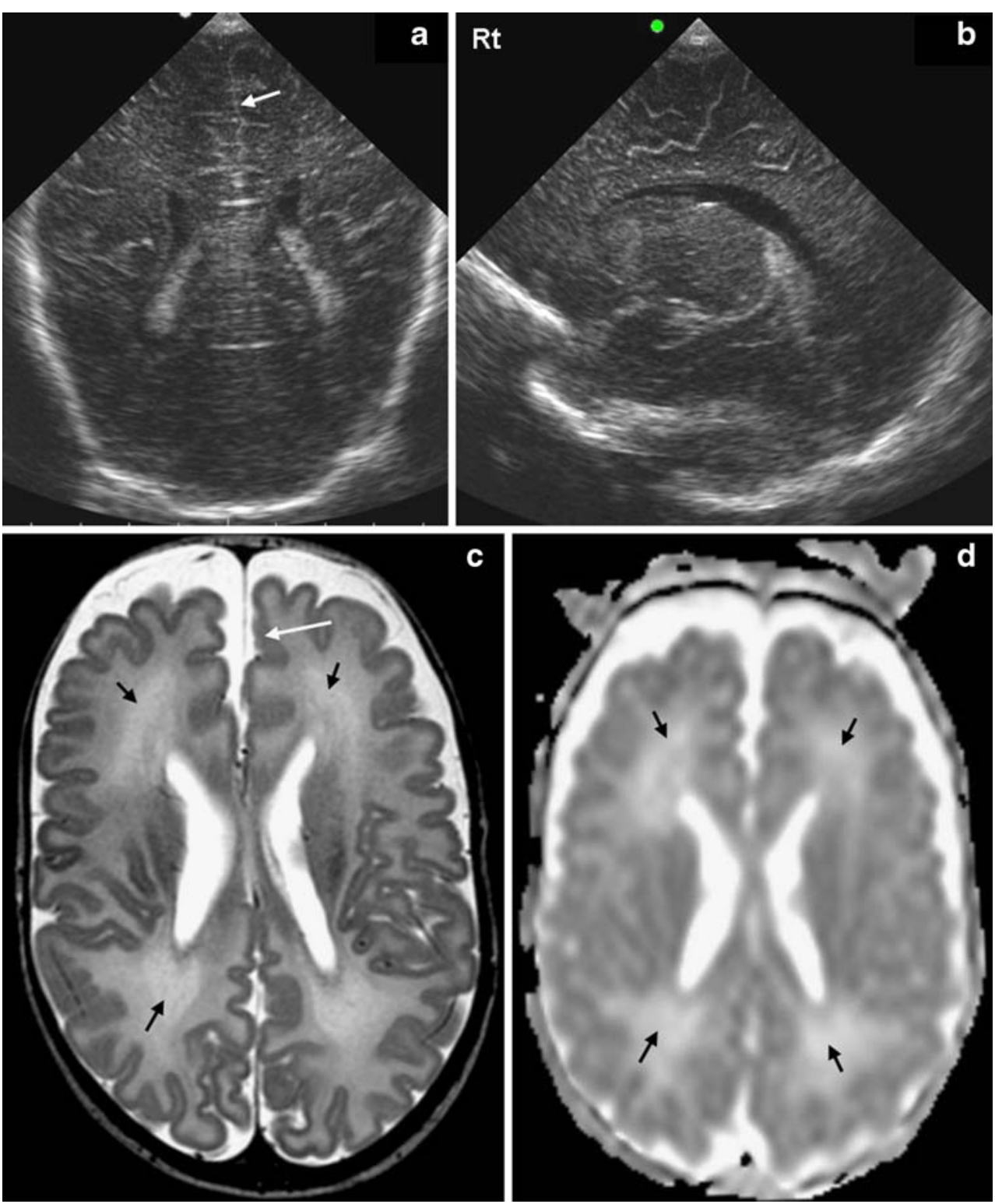

homogeneous echodensities, which is consistent with a previous study [10].

In all five infants with MRI around TEA, DEHSI was present in the WM. DEHSI is found in up to $80 \%$ of preterm infants around TEA and may persist for several weeks $[4,19,27]$. It is associated with cerebral atrophy, WM lesions, and significantly increased diffusivity, suggesting that it represents diffuse WM injury [19, 22, 24, 27]. Infants with DEHSI have a less optimal neurodevelopment than those with normal-appearing WM around TEA $[27,28]$, indicating that DEHSI can be of clinical importance and may be related to the high incidence of neurodevelopmental impairment in preterm infants [29]. So far, no cUS correlate has been established for DEHSI. In our infants with DEHSI, WM changes on cUS were variable. Neurodevelopmental outcome of these infants tended to be suboptimal, which may not only be attribut- able to DEHSI but also to other (WM) abnormalities. From this small group of infants, no conclusions can be drawn about the relation between DEHSI and certain WM changes on cUS and neurodevelopmental outcome.

Table 2 Relation between cUS findings and neurodevelopmental outcome at 2 years corrected age ( $n$, number of infants)

\begin{tabular}{lccc}
\hline $\begin{array}{l}\text { cUS findings } \\
(n=32)\end{array}$ & \multicolumn{3}{l}{ Outcome } \\
\cline { 2 - 4 } & $\begin{array}{l}\text { Normal-mildly } \\
\text { abnormal } \\
(n=20)\end{array}$ & $\begin{array}{l}\text { Moderately } \\
\text { abnormal } \\
(n=5)\end{array}$ & $\begin{array}{l}\text { Severely } \\
\text { abnormal } \\
(n=7)\end{array}$ \\
\hline $\begin{array}{l}\text { Normal-mildly } \\
\text { abnormal }(n=21) \\
\begin{array}{l}\text { Moderately abnormal } \\
(n=7)\end{array}\end{array}$ & 17 & 2 & 2 \\
\begin{tabular}{l} 
Severely abnormal $(n=4)$ \\
\hline
\end{tabular} & 3 & 2 & 2 \\
\hline
\end{tabular}


Table 3 Predictive values of normal to mildly abnormal and severely abnormal cUS and/or MRI findings for, respectively, normal to mildly abnormal and severely abnormal neurodevelopmental outcome at 2 years corrected age

\begin{tabular}{|c|c|c|c|c|c|}
\hline \multirow[t]{2}{*}{ Neuroimaging findings } & & \multicolumn{4}{|c|}{ Predictive values for outcome } \\
\hline & & Sensitivity & Specificity & PPV & NPV \\
\hline \multirow[t]{2}{*}{ cUS findings $(n=32)$} & Normal-mildly abnormal & 0.81 & 0.73 & 0.85 & 0.67 \\
\hline & Severely abnormal & 0.75 & 0.86 & 0.43 & 0.96 \\
\hline \multirow[t]{2}{*}{ MRI findings $(n=32)$} & Normal-mildly abnormal & 0.80 & 0.67 & 0.80 & 0.67 \\
\hline & Severely abnormal & 1.00 & 0.86 & 0.43 & 1.00 \\
\hline \multirow[t]{2}{*}{ cUS and MRI findings $(n=28)$} & Normal-mildly abnormal & 0.79 & 0.78 & 0.88 & 0.64 \\
\hline & Severely abnormal & 1.00 & 0.84 & 0.43 & 1.00 \\
\hline
\end{tabular}

$P P V$ Positive predictive value, $N P V$ negative predictive value

We found severely abnormal WM on cUS to be predictive of adverse neurodevelopmental outcome at 2 years corrected age, while normal or only mildly abnormal WM was predictive of favorable outcome. If the WM was moderately abnormal on cUS, outcome was variable. These results are partially consistent with those from previous studies, indicating that severely abnormal WM on cUS generally predicts adverse outcome $[3,6,7$, 30]. However, in infants with normal WM, cUS has been suggested to be a poor predictor of neurodevelopmental outcome, attributed to the lower sensitivity of cUS for detecting diffuse and more subtle WM injury $[4,5,11,12$, 18, 21, 23]. Differences between those and our studies include the cUS classification of WM changes; we did not consider the total duration of periventricular echodensities but focused on their degree and homogeneity, comparing the echogenicity of the WM to that of the choroid plexus, and relating outcome to the most severe WM changes during admission. Only homogeneous grade 1 echodensities were considered a normal finding. Grade 2 echodensities were classified as moderately abnormal. Also, the frequency and continuation of, and the interval between cUS examinations may differ between studies. We continued cUS throughout the neonatal period until TEA, while others performed cUS less frequently and/or only during the early neonatal period [23].

Several studies have suggested that milder echodensities, if long-lasting, may be associated with suboptimal or deviant neurological development $[2,4-6,13-16]$. Al- though inhomogeneous echodensities seem to be associated with more severe WM changes on MRI [10], these studies did not make a distinction in appearance of echodensities. Of the 11 infants with homogeneous grade 1 echodensities for whom outcome data were available, 10 were normal or mildly abnormal (7 normal, 3 mildly abnormal), and only one was severely abnormal at 2 years. This latter infant showed bilateral intraventricular hemorrhages grade 3 with posthemorrhagic ventricular dilatation on neonatal cUS and MRI, which probably explains his severely abnormal outcome. We therefore hypothesize that homogeneous grade 1 echodensities represent normal maturational phenomena in the immature brain, especially if occurring in the frontal or parietal WM $[9,17]$.

Like cUS, severely abnormal WM on MRI within the first three postnatal months was highly predictive of adverse outcome, while normal or only mildly abnormal WM predicted a favorable outcome in almost all cases. Moderately abnormal WM on MRI was associated with variable outcome. Previous studies have shown a good correlation between WM changes as detected on MRI and outcome [3, 30, 31]. In our study, moderately abnormal WM on MRI was associated with more variable outcome than in other studies. This may be related to the timing and the variance in timing of the MRI examinations. During the study period, MRI in preterm infants was still done before discharge or transfer to another hospital, so mostly before TEA. Nowadays, it is preferably performed around TEA. DEHSI, possibly associated with less favorable outcome, is

Table 4 Relation between MRI findings and neurodevelopmental outcome at 2 years corrected age

\begin{tabular}{lccc}
\hline MRI findings $(n=32)$ & Outcome & & \\
\cline { 2 - 4 } & Normal-mildly abnormal $(n=20)$ & Moderately abnormal $(n=5)$ & Severely abnormal $(n=7)$ \\
\hline Normal-mildly abnormal $(n=20)$ & 16 & 2 & 2 \\
Moderately abnormal $(n=9)$ & 4 & 3 & 2 \\
Severely abnormal $(n=3)$ & & & 3 \\
\hline
\end{tabular}

$n$ Number of infants 
Table 5 Relation between cUS and MRI findings and neurodevelopmental outcome at 2 years corrected age

\begin{tabular}{lccc}
\hline cUS and MRI findings $(n=28)$ & \multicolumn{2}{l}{ Outcome } & \\
\cline { 2 - 4 } & $\begin{array}{l}\text { Normal-mildly } \\
\text { abnormal }(n=17)\end{array}$ & $\begin{array}{l}\text { Moderately } \\
\text { abnormal }(n=4)\end{array}$ & $\begin{array}{l}\text { Severely abnormal } \\
(n=7)\end{array}$ \\
\hline Normal-mildly abnormal $(n=19)$ & 15 & 2 & 2 \\
Moderately abnormal $(n=6)$ & 2 & 2 & 3 \\
Severely abnormal $(n=3)$ & & & 3 \\
\hline
\end{tabular}

$n$ Number of infants

WM changes, we feel that this would not have influenced our findings considerably. Secondly, we evaluated the most severe cUS findings and the MRI findings obtained at different ages, and not cUS and MRI findings obtained on the same day. This may limit the reliability of the MRI and its predictive value for neurodevelopmental outcome. Thirdly, we only obtained neuroimaging data around TEA in a few infants and were therefore not able to assess brain growth and maturation properly. In only five infants, MRI was performed around TEA, which is probably the most optimal time for MR imaging in preterm infants $[3,4,19$, $27,30,31]$. There was a substantial variability in postnatal age at MRI scanning. This may have influenced our results; it is possible that some abnormalities (such as SI changes) are best seen on early diffusion-weighted scans, while others (such as cystic lesions) need time to develop and are therefore better or only recognized on scans performed at older age. However, no differences in predictive values of MRI findings for outcome were found between the infants scanned at a very young postnatal age $(<10$ days) and those scanned later ( $\geq 10$ days). And there was no tendency of severe lesions occurring more often in the infants scanned at older age ( $\geq 10$ days) than in the infants scanned at very young age $(<10$ days). Another possible limitation is that our study was retrospective and performed in a relatively small number of infants. Because of the small number of infants, we did not assess a possible relation between the site and shape of the WM changes and outcome [15, 35]. However, comparable data have been obtained in a prospective study in preterm infants [12]. Finally, not in all infants Bayley II scores or neurodevelopmental outcome data at 2 years were available, and outcome was assessed at a relatively young age, so, developmental problems may still occur in these infants. A larger, prospective study with longer-term follow-up is needed to analyze the relation between neuroimaging findings and neurodevelopmental outcome in more detail.

In conclusion, this study shows that in very preterm infants, sequential, high-quality cUS throughout the neonatal period is a reliable tool for detecting WM changes. Homogeneous grade 1 echodensities on cUS probably represent normal (maturational) phenomena in the preterm brain and inhomogeneous grade 1 echodensities possibly reflect punctate WM lesions. cUS is predictive of favorable duration of echodensities between the different groups of 
and severely abnormal outcome at 2 years corrected age. MRI within the first three postnatal months is only of clinical importance for outcome prediction in infants with severe WM changes on cUS. So, conventional and diffusion-weighted MRI sequences before TEA in very preterm infants, suggested on cUS to have mild to moderately abnormal WM, do not seem to be warranted, and a combination of sequential cUS and a MRI around TEA probably provides more valuable information and is more predictive of neurodevelopmental outcome.

Conflict of interest statement We declare that we have no conflict of interest.

Open Access This article is distributed under the terms of the Creative Commons Attribution Noncommercial License which permits any noncommercial use, distribution, and reproduction in any medium, provided the original author(s) and source are credited.

\section{References}

1. de Vries LS (1996) Neurological assessment of the preterm infant. Acta Paediatr 85:765-771

2. de Vries LS, Eken P, Dubowitz LM (1992) The spectrum of leukomalacia using cranial ultrasound. Behav Brain Res 49:1-6

3. Roelants-van Rijn AM, Groenendaal F, Beek E et al (2001) Parenchymal brain injury in the preterm infant: comparison of cranial ultrasound, MRI and neurodevelopmental outcome. Neuropediatrics 32:80-89

4. Maalouf EF, Duggan PJ, Counsell SJ et al (2001) Comparison of findings on cranial ultrasound and magnetic resonance imaging in preterm infants. Pediatrics 107:719-727 DOI 10.1542/ peds.107.4.719

5. Miller SP, Cozzio CC, Goldstein RB et al (2003) Comparing the diagnosis of white matter injury in premature newborns with serial MR imaging and transfontanel ultrasonography findings. AJNR Am J Neuroradiol 24:1661-1669

6. de Vries LS, van Haastert IL, Rademaker KJ et al (2004) Ultrasound abnormalities preceding cerebral palsy in high-risk preterm infants. J Pediatr 144:815-820 DOI 10.1016/j. jpeds.2004.03.034

7. Rademaker KJ, Uiterwaal CSPM, Beek FJA et al (2005) Neonatal cranial ultrasound versus MRI and neurodevelopmental outcome at school age in children born preterm. Arch Dis Child Fetal Neonatal Ed 90:F489-F493 DOI 10.1136/adc.2005.073908

8. van der Knaap MS, van Wezel-Meijler G, Barth PG et al (1996) Normal gyration and sulcation in preterm and term neonates: appearance on MR images. Radiology 200:389-396

9. van Wezel-Meijler G, van der Knaap MS, Sie LTL et al (1998) Magnetic resonance imaging of the brain in premature infants during the neonatal period. Normal phenomena and reflection of mild ultrasound abnormalities. Neuropediatrics 29:89-96

10. Sie LTL, van der Knaap MS, van Wezel-Meijler G et al (2000) Early MR features of hypoxic-ischemic brain injury in neonates with periventricular densities on sonograms. AJNR Am J Neuroradiol 21:852-861

11. Debillon T, N'Guyen S, Muet A et al (2003) Limitations of ultrasonography for diagnosing white matter damage in preterm infants. Arch Dis Child Fetal Neonatal Ed 88:F275-F279 DOI 10.1136/fn.88.4.F275

12. Inder TE, Anderson NJ, Spencer C et al (2003) White matter injury in the premature infant: a comparison between serial cranial sonographic and MR findings at term. AJNR Am J Neuroradiol 24:805-809

13. Jongmans M, Henderson S, de Vries L et al (1993) Duration of periventricular densities in preterm infants and neurological outcome at 6 years of age. Arch Dis Child 69:9-13

14. van Wezel-Meijler G, van der Knaap MS, Oosting J et al (1999) Predictive value of neonatal MRI as compared to ultrasound in premature infants with mild periventricular white matter changes. Neuropediatrics 30:231-238

15. Resch B, Jammernegg A, Perl E et al (2006) Correlation of grading and duration of periventricular echodensities with neurodevelopmental outcome in preterm infants. Pediatr Radiol 36:810-815 DOI 10.1007/s00247-006-0178-2

16. Kutschera J, Tomaselli J, Maurer U et al (2006) Minor neurological dysfunction, cognitive development and somatic development at the age of 3 to 11 years in very-low-birthweight infants with transient periventricular echodensities. Acta Paediatr 95:1577-1581 DOI 10.1080/08035250600643236

17. Boxma A, Lequin M, Ramenghi LA et al (2005) Sonographic detection of the optic radiation. Acta Paediatr 94:1455-1461 DOI 10.1111/j.1651-2227.2005.tb01820.x

18. Paneth N, Rudelli R, Monte W et al (1990) White matter necrosis in very low birth weight infants: neuropathologic and ultrasonographic findings in infants surviving six days or longer. J Pediatr 16:975-984

19. Maalouf EF, Duggan PJ, Rutherford MA et al (1999) Magnetic resonance imaging of the brain in a cohort of extremely preterm infants. J Pediatr 135:351-357

20. Hüppi PS, Murphy B, Maier SE et al (2001) Microstructural brain development after perinatal cerebral white matter injury assessed by diffusion tensor magnetic resonance imaging. Pediatrics 107:455-460 DOI 10.1542/peds.107.3.455

21. Childs AM, Cornette L, Ramenghi LA et al (2001) Magnetic resonance and cranial ultrasound characteristics of periventricular white matter abnormalities in newborn infants. Clin Radiol 56:647-655 DOI 10.1053/crad.2001.0754

22. Counsell SJ, Allsop JM, Harrison MC et al (2003) Diffusionweighted imaging of the brain in preterm infants with focal and diffuse white matter abnormality. Pediatrics 112:1-7 DOI 10.1542/peds. 112.1 .1

23. Mirmiran M, Barnes PD, Keller K et al (2004) Neonatal brain magnetic resonance imaging before discharge is better than serial cranial ultrasound in predicting cerebral palsy in very low birth weight preterm infants. Pediatrics 114:992-998 DOI 10.1542/ peds.2003-0772-L

24. Counsell SJ, Shen Y, Boardman JP et al (2006) Axial and radial diffusivity in preterm infants who have diffuse white matter changes on magnetic resonance imaging at term-equivalent age. Pediatrics 117:376-386 DOI 10.1542/peds.2005-0820

25. Volpe JJ (1995) Intracranial hemorrhage: germinal matrix-intraventricular hemorrhage of the premature infant. In: Volpe JJ (ed) Neurology of the newborn. Saunders, Philadelphia, pp 403-463

26. Bayley N (1993) Bayley scales of infants development, 2nd edn. Psychological Corporation, San Antonio, TX

27. Dyet LE, Kennea N, Counsell SJ et al (2006) Natural history of brain lesions in extremely preterm infants studied with serial magnetic resonance imaging from birth and neurodevelopmental assessment. Pediatrics 118:536-548 DOI 10.1542/peds.2005-1866

28. Domizio S, Barbante E, Puglielli C et al (2005) Excessively high magnetic resonance signal in preterm infants and neuropsychobehavioural follow-up at 2 years. Int J Immunopathol Pharmacol 18:365-375 
29. Marlow N (2004) Neurocognitive outcome after very preterm birth. Arch Dis Child Fetal Neonatal Ed 89:F224-F228 DOI 10.1136/adc.2002.019752

30. Woodward LJ, Anderson PJ, Austin NC et al (2006) Neonatal MRI to predict neurodevelopmental outcomes in preterm infants. N Engl J Med 355:685-694

31. Valkama AM, Paakko EL, Vainionpaa LK et al (2000) Magnetic resonance imaging at term and neuromotor outcome in preterm infants. Acta Paediatr 89:348-355 DOI 10.1080/0803525007 50028519

32. Hüppi PS, Warfield S, Kikinis R et al (1998) Quantitative magnetic resonance imaging of brain development in premature and mature newborns. Ann Neurol 43:224-235
33. Ajayi-Obe M, Saeed N, Cowan FM et al (2000) Reduced development of cerebral cortex in extremely preterm infants. The Lancet 356:1162-1163 DOI 10.1016/S0140-6736(00)02761-6

34. Inder TE, Warfield SK, Wang $H$ et al (2005) Abnormal cerebral structure is present at term in premature infants. Pediatrics 115:286-294 DOI 10.1542/peds.2004-0326

35. Rademaker KJ, Groenendaal F, Jansen GH et al (1994) Unilateral haemorrhagic parenchymal lesions in the preterm infant: shape, site and prognosis. Acta Paediatr 83:602-608

36. Leijser LM, Klein RH, Veen S et al (2004) Hyperechogenicity of the thalamus and basal ganglia in very preterm infants: radiological findings and short-term neurological outcome. Neuropediatrics 35:283-289 DOI 10.1055/s-2004-830364 